\title{
Pertinent Shape Effects of Gold Nanospheres and Nanostellates
}

\author{
Manoj Verma, ${ }^{1}$ Boazbou Newmai, ${ }^{1}$ Abhitosh Kedia ${ }^{2}$ and P. Senthil Kumar ${ }^{1 *}$ \\ ${ }^{1}$ Department of Physics \& Astrophysics, University of Delhi, Delhi-110007, India \\ ${ }^{2}$ Department of Physics, Uka Tarsadia University, Bardoli, Surat-394350, Gujarat, India
}

\begin{abstract}
Modulating optical resonances in plasmonic resonators were easily achieved by virtue of their constituent composition, size as well as shape. Such confinement of electric field in the nano-regime enhances extra-ordinarily the light-matter interaction, further enabling wide range of applications in nanophotonics, SERS etc. Herein, we compare and contrast the surface plasmon modes of Au nanospheres and nanostellates, the visibly distinct morphologies of which not only correlates exceptionally well with their structure-property functional relationships, but also articulate their respective growth mechanisms in a rather meticulous manner. Higher amplification of analyte Raman signals visibly demonstrates the increased number of hot-spots with reference to their surface morphology and roughness.
\end{abstract}

Keywords: Shape evolution, Plasmonic resonances, X-ray diffraction, SERS.

\section{Introduction}

In metal nanostructures, the coupling of incident light to resonant motion of the free electron plasma (known as plasmons) allows confinement, control and enhancement of electromagnetic fields at nanometer scale.[1] The formation of such plasmon-based structures has gained tremendous momentum and inspired scientist for promising research activities ranges from nanoscale antennas to photovoltaics, or plasmonic waveguiding. Furthermore, the high local field enhancements in the close proximity of metal nanoparticles can be subjugated for sensing or for Surface Enhanced Raman Scattering (SERS).[2]

Plasmonic nanostructures endowed with sharp tips or edges ascertain the best possible substrates for SERS compared to spherical geometry for significant molecule detection. $[3,4]$ The 3D architecture, the sharp tips of the nanostars promote the formation of localized giant electric-field.[5] Systematic experiments and theoretical simulation imply that these 3D non-oriented planner tips configuration permits multiple hot spots for improving SERS performance compared to planar counterparts with comparable plasmon resonance position.

In this report, we have seen that hydrogen bonding/dipole interactions arising due to mixing of PVP with protic/aprotic polar solvents such as water/ methanol and DMF leads to drastic change in conformation behavior of polymer in nucleation regime and along with metal ions can direct to interesting optical/plasmonic properties of formed metal nanoparticles.[6] Importance of nanocrystallite shape on structure correlated optical properties along with crystal diffraction parameters is studied carefully. Further, in the case of pristine gold nanostellates, the EM field amplification due to high local curvature results in attractive SERS signals.

\section{Experimental}

In a typical synthesis of gold nanospheres, $0.27 \mathrm{mM}$ aqueous solution of hydrochloroauric acid $\left(\mathrm{HAuCl}_{4} \cdot 3 \mathrm{H}_{2} \mathrm{O}\right)$ was mixed with $15 \mathrm{~mL}$ of $10 \mathrm{mM}$ polyvinylpyrrolidone (PVP average $\left.\mathrm{MW}=10,000\right)$ solution in methanol at room temperature. Nanostellates were synthesized by mixing $(0.27 \mathrm{mM})$ aqueous solution of $\mathrm{HAuCl}_{4} \cdot 3 \mathrm{H}_{2} \mathrm{O}$ with $15 \mathrm{~mL}$ of $10 \mathrm{mM}$ PVP solution in DMF. In both cases transformation from pale yellow solution to vibrant colors within few hours of reaction signifies the formation of nanoparticles.

Optical absorption measurements were carried out in the wavelength range of $200-1100 \mathrm{~nm}$ using Thermo Scientific absorption spectrophotometer. TEM images were acquired using the FE-Technai G2 system operated at an accelerating voltage of $300 \mathrm{kV}$. The crystal structure parameters ware evaluated by analysing the X-ray diffraction (XRD) spectra collected with $\mathrm{Cu}-\mathrm{K} \alpha$ radiation using a Bruker D-8 Advance X-ray diffractometer. The surface enhanced Raman scattering (SERS) spectra were obtained using a Renishaw inVia Raman spectrometer with $785 \mathrm{~nm} \mathrm{He}-\mathrm{Ne}$ laser source.

\section{Results And Discussion}

Absorption Spectra and TEM micrographs of Nanospheres and Nanostellates:

Universal solvent adaptable homogenous polymer, Polyvinylpyrrolidone (PVP), was effectively utilized in fine tuning the nucleation and growth parameters, resulting in hyper-branched 3D gold nanostructures (nanostellates) in DMF solvent, whereas yielding the isotropic spherical gold nanostructures in methanol, for the fixed PVP to metal ion ratio. 
The unique optical properties of different shaped $\mathrm{Au}$ nanocrystals were visibly observed by the distinct color of their colloidal solutions (red color in methanol solvent and bluish color in DMF, see the inset photos in figure 1a), thereby corresponding to single SPR peak at $2.33 \mathrm{eV}$ in case of na nospheres due to the symmetrical distribution of plasmons over the entire shape, while the nanostellates show non-uniform dual modes at 2.13 and $1.37 \mathrm{eV}$, pertaining to the plasmon oscillations w.r.t. core and the spikes respectively, as demonstrated from their corresponding TEM images in figures $1 \mathrm{~b}-\mathrm{d}$.

Low energy SP resonance in case of Au nanostellates were straightforwardly attributed to the large electric field confinement supported onto their highly anisotropic tips. As each of the nanostellate tips have their own highly individual plasmon oscillations (meaning, higher local density of states), their asymmetrically oriented complex architecture couple the nearby tips via their dynamically strong near-field interaction. As a result, the unusual intra-plasmon hybridization arises due to the intense presence of different non-planar oriented tips, thereby broadening as well as inclining the resonance peak around $2.13 \mathrm{eV}$.

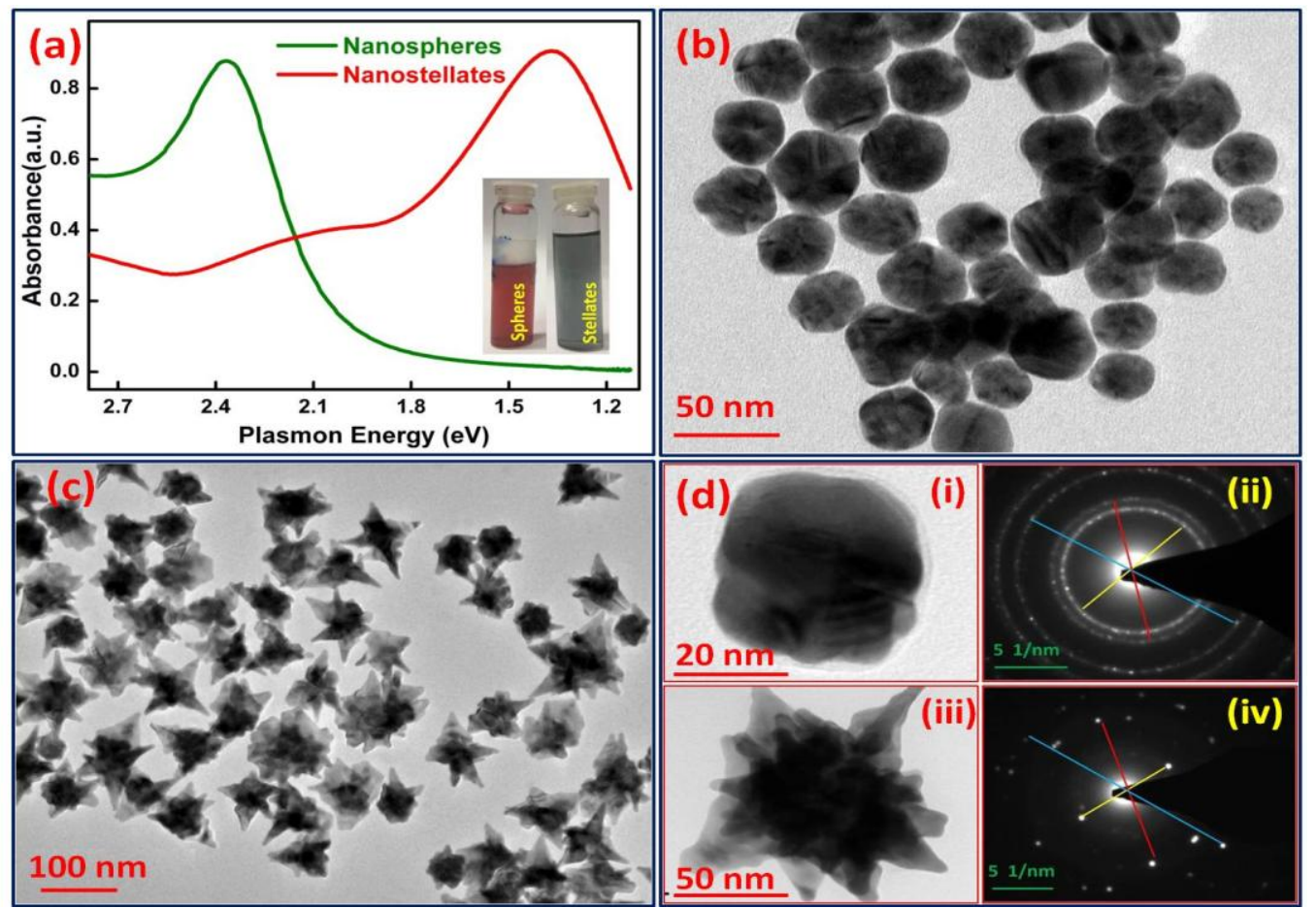

Fig. 1 (a) The SPR spectra of Au nanospheres and nanostellates in the visible-infrared energy range, TEM images of Au nanospheres (b) and nanostellates (c) and their corresponding HR single particle image and SAED patterns are shown in $(\mathrm{d})$.

In general, final size of nanoparticles synthesized depend on the nucleation time while anisotropic shape arises due to slow growth kinetics which can be supported by nucleation growth kinetics of nanospheres and nanostellates. Early nucleation in case of nanospheres and slow growth in case of nanostellates beautifully explains the size distribution histogram for both types of nanoparticles synthesized (Figure 2).
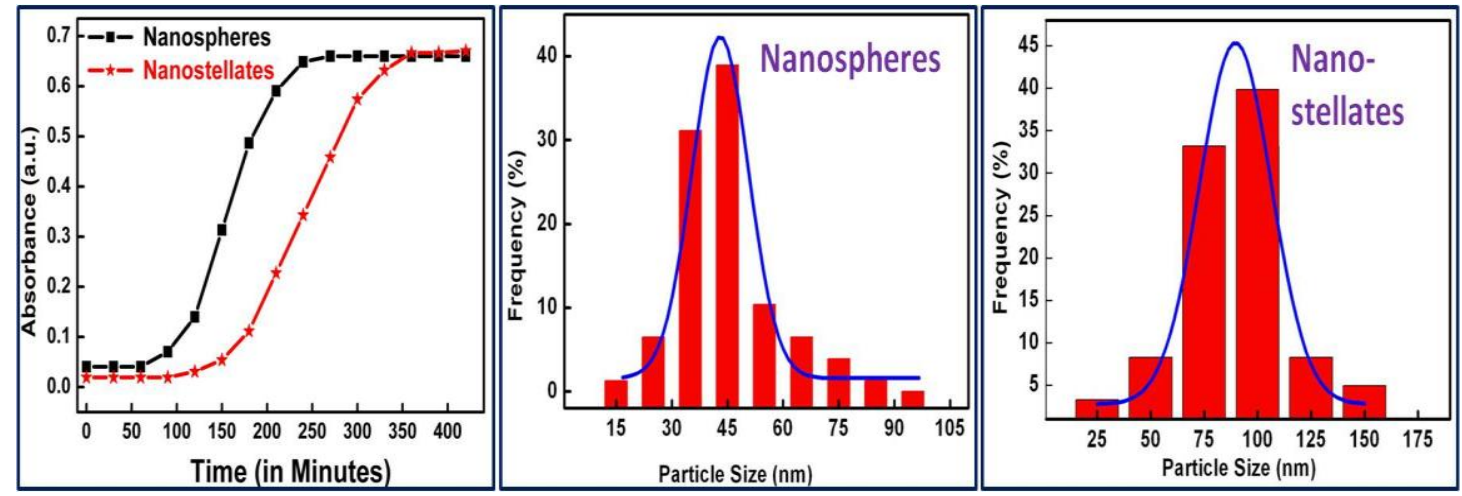

Fig. 2 Absorption kinetics during formation of spherical and star shaped nanoparticles and their corresponding final size and monodispersity histogram. 
HR-TEM images for the single Au nanoparticles are shown in Fig. 1(d), clearly contrasting their varied intriguing morphology, which corroborates very well with the optical absorption spectra given in Fig. 1a. Selected area electron diffraction (SAED) pattern provides intuitive information on the extent of crystallinity of the nanoparticles.

\begin{tabular}{|c|c|c|c|c|c|c|c|c|c|}
\hline \multirow{2}{*}{$\begin{array}{l}\text { Shape of } \\
\text { Nanoparticle }\end{array}$} & \multicolumn{3}{|c|}{ Yellow Ring <111> } & \multicolumn{3}{|c|}{ Red Ring <200> } & \multicolumn{3}{|c|}{ Cyan Ring <220> } \\
\hline & $2 \mathrm{R}(1 / \mathrm{nm})$ & $\mathrm{R}(1 / \mathrm{nm})$ & "d" (nm) & $2 \mathrm{R}(1 / \mathrm{nm})$ & $\mathrm{R}(1 / \mathrm{nm})$ & "d" (nm) & $2 \mathrm{R}(1 / \mathrm{nm})$ & $\mathrm{R}(1 / \mathrm{nm})$ & "d" (nm) \\
\hline Nanospheres & 8.55 & 4.27 & 0.234 & 9.85 & 4.92 & 0.203 & 13.99 & 6.99 & 0.143 \\
\hline Nanostellates & 8.42 & 4.21 & 0.237 & 9.60 & 4.80 & 0.208 & 13.68 & 6.84 & 0.146 \\
\hline
\end{tabular}

Table 1 Measured diameter 2R, $\mathrm{R}$ in reciprocal space and corresponding d value in real space for nanospheres and nanostellates respectively.

While the diffuse diffraction rings consistently characterize the overall polycrystalline nature of the asformed $\mathrm{Au}$ nanostructures, the bright spots on each ring correspond to a particular crystallographic facet. The lattice parameter values obtained from the first three consecutive rings (yellow, red and cyan) from the centre (see Table 1) corresponds to $\langle 111\rangle,\langle 200\rangle$ and $\langle 220\rangle$ facets respectively. In case of Au nanospheres, a large number of bright spots appear in each ring, assimilating the highly in-planar yet random distribution of crystallites in various crystallographic planes, whereas in case of Au nanostellates, the higher intensity with lesser number of bright spots suggest the non-planar as well as omni-directional orientation of the spikes/tips, indicative of their unique, 3D anisotropic growth.[5]

X-Ray diffraction studies revealing the differential crystal structures in Nanospheres and Nanostellates: XRD patterns of the synthesized Au nanostructures, shown in Fig. 3(a), reveal evidently their high crystalline nature, with subtle differences in the peak positions (see Table 2).

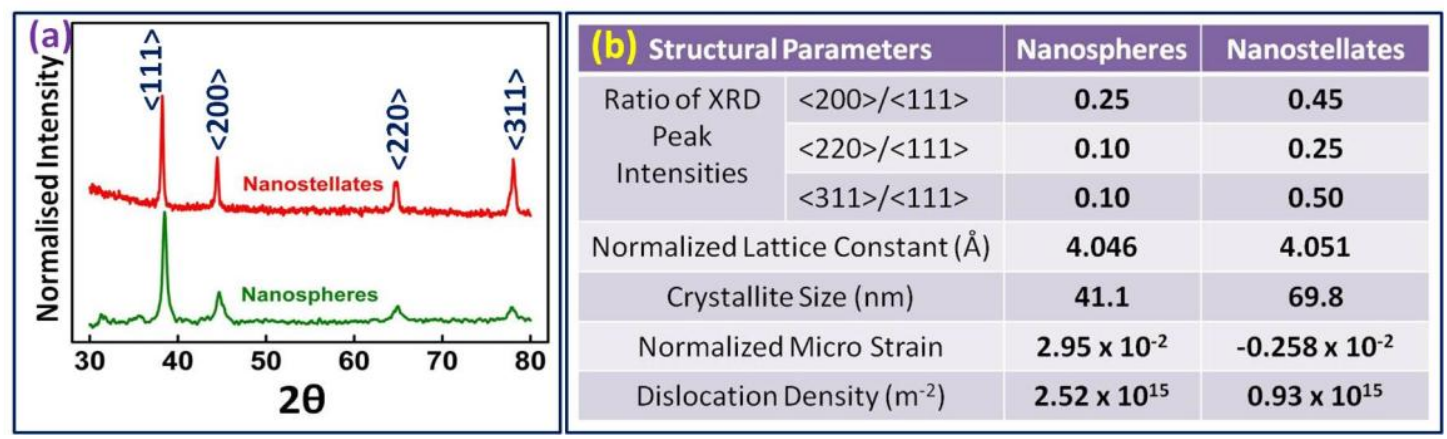

Fig. 3 X-ray diffraction pattern of spherical and star nanoparticles (a) and calculated structural parameters for both types of nanocrystals (b).

In case of Au nanospheres, the XRD peaks are broadened due to their nanocrystallite size regime, while for the Au nanostellates, they are sharper with minute blue-shift in their peak positions, corresponding to the induced micro-strain due to the compact constellation of various spikes/tips, right from their growth stage itself.[7] The normalized ratio of peak intensities in comparison with the <111> peak in case of Au nanostellates (as calculated and tabulated in Fig. 3b) indicates the larger crystallite size, slow growth kinetics (as deposition on high energy crystal facet is slow) and anisotropic shape evolution as compared to that of Au nanospheres.

\begin{tabular}{|l|l|l|l|}
\hline Shapes of Nanoparticles & Planes & $2 \theta$ (in Degrees) & Intensity \\
\hline \multirow{5}{*}{ Nanospheres } & $<111>$ & 38.4025 & 100 \\
\cline { 2 - 4 } & $<200>$ & 44.6528 & 25 \\
\cline { 2 - 4 } & $<220>$ & 64.7920 & 10 \\
\cline { 2 - 4 } & $<311>$ & 77.9935 & 10 \\
\hline Nanostellates & $<111>$ & 38.2516 & 100 \\
\cline { 2 - 4 } & $<200>$ & 44.4050 & 45 \\
\cline { 2 - 4 } & $<220>$ & 64.8955 & 25 \\
\cline { 2 - 4 } & $<311>$ & 78.0936 & 50 \\
\hline
\end{tabular}

Table 2 Diffraction peak positions and their normalized peak intensities for Au nanospheres and nanostellates respectively.

Furthermore, the decrease in microstrain with increasing NRF indicates compressive nature of strain due to which preferential growth on low energy facets takes place (as in Fig. 3b and Figure 4), thus signifying the formation of isotropic Au nanospheres. On the other hand, the increasing microstrain with NRF indicates its 
tensile nature which initiates growth along high energy facets necessary for the anisotropic shape evolution as in the case of $\mathrm{Au}$ nanostellates, visualizing the complex cris-crossing of relatively larger anisotropic crystallites into a compact nanostructure. Moreover, as seen from Fig. 3(b) and Figure 4, the expected lattice constant in these two dissimilar Au nanostructures changes due to different nature of strain present and thus overall stress exerted on their unit cell changes.
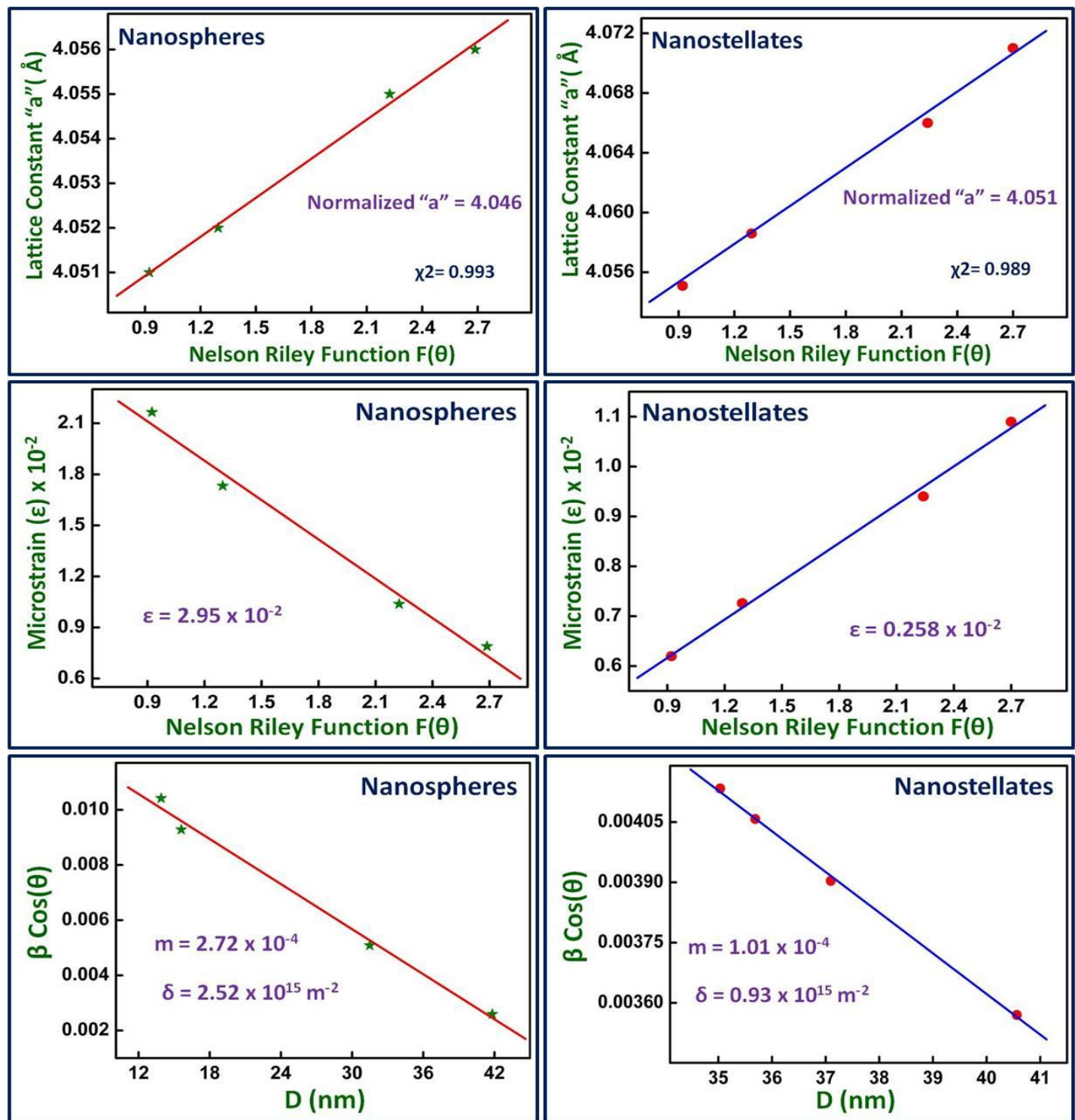

Fig. 4 Plots of "a" value and Microstrain versus Nelson riley function for spherical and star shaped nanoparticles. Dislocation density for both particles was calculated by plotting $\beta \cos (\theta)$.

In case of Au nanospheres, due to smaller crystallite size and thus relatively large number of crystallite per unit volume, the presence of grain/tilt boundaries and stacking faults within the nanoparticle were found to be abundant. These cumulative surface and volume effects enhance the dislocation density of Au nanospheres in direct contrast with that of the Au nanostellates (see Fig. 3b and Figure 4), thereby meticulously correlating the aesthetics on the structure-property functional relationships.

\section{SERS capabilities of Nanospheres and Nanostellates:}

The enhanced SERS aspects of the Au nanostructures were studied using the analyte crystal violet. In brief, a monolayer of PVP coated gold nanostructures (serving as plasmonic substrates) was carefully formed on a silicon substrate. 


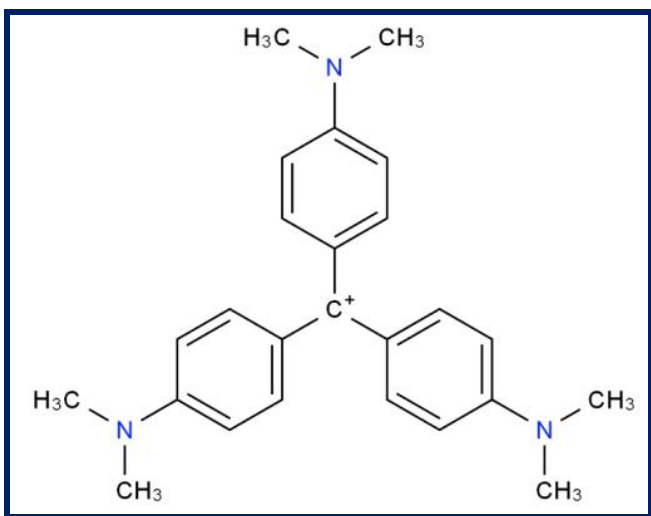

Fig. 5 Structure of Crystal violet dye molecule used for investigating SERS capabilities of synthesized nanoparticles.

For crystal violet, various peaks in raman shift ranges from 200 to $1700 \mathrm{~cm}^{-1}$ arises due to central carbon atoms, nitrogen atoms and $\pi$ electrons in the phenyl ring of the dye molecule (see Fig. 5). Though the concentration $(1 \mu \mathrm{M})$ of $\mathrm{CV}$ molecules and the excitation wavelength $(785 \mathrm{~nm})$ of laser used was kept constant for both shapes, the significant difference in intensity of the Raman signals can be ascribed to their shape only.

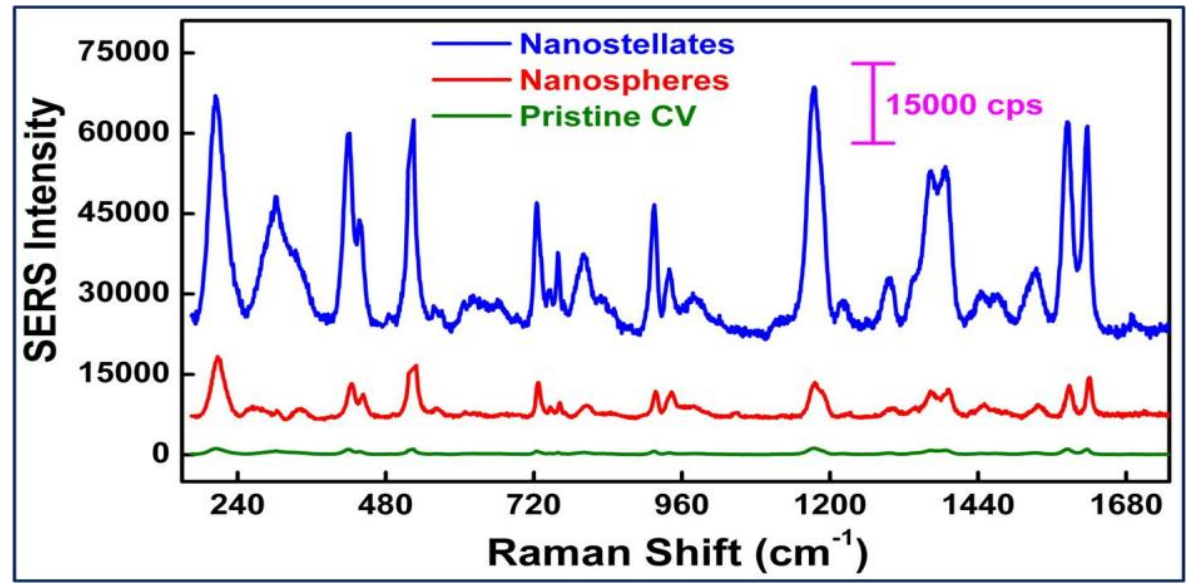

Fig. 6 Raman spectra of crystal violet, pristine and after adsorption on Au nanospheres and nanostellates.

In comparison with gold nanostellates, the SERS signal intensity of the adsorbed CV dye molecules were found to be typically weak in case of nanospheres as seen in Fig. 6. Clearly, raman intensity of even $1 \mu \mathrm{M}$ of analyte increases by a factor of around $1.5 \times 10^{4}$ and $1.67 \times 10^{5}$ in case of Au nanospheres and nanostellates respectively, in comparison with the raman signal of a higher concentration $(1 \mathrm{mM})$ of the pristine dye.

The SERS enhancement due to chemisorption were found to be negligible in both our present cases, as there was neither a red-shift nor a blue-shift of analyte vibration peaks.[8] Thus, the observed tremendous SERS enhancement was clearly attributed to increased photonic density of states in case of Au nanostellates. Moreover, as revealed from the diffraction studies, larger crystallite domain extended along high energy facets drastically affect the plasmon dephasing and hence boost the characteristic electromagnetic enhancement effects.

Based on above results, it is conclusive that the electric field enhancement at the spikes/tips and clearly a large number of hot-spots were available in the nanostellate type geometry. An enhanced charge density localization near the pointed tips of these nanostructures, popularly known as lightning rod effect, allows the Au nanostellates to act as better optical antenna than the corresponding Au nanospheres.

\section{Conclusions}

Tuning the PVP polymer reducing/capping strengths resulted in different shape evolution of the Au nanostructures even under ambient conditions. The diffraction studies revealed the crystallization process involved in differential shape evolution. SERS results imply that asymmetric distribution of LDOS within the single Au nanostar induced by hybridization of the strong intra-particle plasmonic coupling; due to the presence of the numerous asymmetrical nonplanar tips oriented in random direction can serve as a promising configuration for enhanced spectroscopic applications. 


\section{Acknowledgements}

Authors thank University of Delhi for the annual R\&D grant (RC/2015/9677). Authors also thank USIC-DU, SAIF AIIMS for material characterization.

\section{References}

[1]. C. Noguez, Surface Plasmons on Metal Nanoparticles:The Influence of Shape and Physical Environment, The Journal of Physical Chemistry C 111(10) (2007) 3806-3819.

[2]. M. Das, K.H. Shim, S.S.A. An, D.K. Yi, Review on gold nanoparticles and their applications, Toxicology and Environmental Health Sciences 3(4) (2012) 193-205.

[3]. C.G. Khoury, T. Vo-Dinh, Gold Nanostars For Surface-Enhanced Raman Scattering: Synthesis, Characterization and Optimization, The journal of physical chemistry. C, Nanomaterials and interfaces 2008(112) (2008) 18849-18859.

[4]. M. Verma, A. Kedia, M.B. Newmai, P.S. Kumar, Differential role of PVP on the synthesis of plasmonic gold nanostructures and their catalytic and SERS properties, RSC Advances 6(83) (2016) 80342-80353.

[5]. W. Niu, Y.A.A. Chua, W. Zhang, H. Huang, X. Lu, Highly Symmetric Gold Nanostars: Crystallographic Control and SurfaceEnhanced Raman Scattering Property, Journal of the American Chemical Society 137(33) (2015) 10460-10463.

[6]. T.C. Deivaraj, N.L. Lala, J.Y. Lee, Solvent-induced shape evolution of PVP protected spherical silver nanoparticles into triangular nanoplates and nanorods, Journal of Colloid and Interface Science 289(2) (2005) 402-409.

[7]. K.Y. Lee, M. Kim, J.-S. Noh, H.C. Choi, W. Lee, Novel surfactant-free multi-branched gold stars characterized by inverse photocurrent, Journal of Materials Chemistry A 1(44) (2013) 13890-13895.

[8]. S. Chatterjee, A.B. Ringane, A. Arya, G.M. Das, V.R. Dantham, R. Laha, S. Hussian, A high-yield, one-step synthesis of surfactantfree gold nanostars and numerical study for single-molecule SERS application, J Nanopart Res 18(242) (2016) 1-9. 